\title{
ELUCIDANDO OS ESTADOS DE OXIDAÇÃO DO NITROGÊNIO ATRAVÉS DA ESPECTROSCOPIA DE ABSORÇÃO DE RAIOS-X NA BORDA K DO NITROGÊNIO
}

\author{
Gustavo M. do Nascimento* e Marcia L. A. Temperini \\ Departamento de Química Fundamental, Instituto de Química, Universidade de São Paulo, CP 26077, \\ 05513-970 São Paulo - SP, Brasil
}

Recebido em 30/3/05; aceito em 9/9/05; publicado na web em 14/3/06

\begin{abstract}
NITROGEN OXIDATION STATES ELUCIDATED BY X-RAY ABSORPTION NITROGEN K-EDGE SPECTROSCOPY. The potentialities of X-ray Absorption Near Edge Spectroscopy (XANES) of the N K edge (N K) obtained with the spherical grating monochromator beam line at the Brazilian National Synchrotron Light Laboratory are explored in the investigation of poly(aniline), nanocomposites and dyes. Through the analysis of N K XANES spectra of conducting polymers and many other dye compounds that are dominated by $1 \mathrm{~s} \rightarrow \pi *$ transitions, it was possible to correlate the band energy value with the nitrogen oxidation states. An extensive N K XANES spectral database was obtained, thus permitting the elucidation of the nature of different nitrogens present in the intercalated conducting polymers.
\end{abstract}

Keywords: N K XANES; poly(aniline); dyes.

\section{INTRODUÇÃO}

Técnicas experimentais que utilizam radiação eletromagnética no estudo da estrutura da matéria há muito se mostraram de grande importância em várias áreas da química. Por ex., com energia na região do infravermelho podemos sondar as vibrações das moléculas e assim determinar sua estrutura molecular, ou utilizando radiação UV e visível podemos investigar a estrutura eletrônica das mesmas. Mais recentemente o emprego de técnicas que utilizam radiação eletromagnética com energia que vai do UV de vácuo ( 10-40 $\mathrm{eV}, 125-31 \mathrm{~nm})$, passando pelos raios-X moles (40-1500 eV, 31-0,8 $\mathrm{nm})$, e indo até os raios- $\mathrm{X}$ duros $\left(1500-10^{5} \mathrm{eV}, 0,8-0,01 \mathrm{~nm}\right)^{1}$ tem se mostrado de grande importância na elucidação da estrutura eletrônica de diferentes tipos de materiais. Uma das maiores dificuldades para desenvolvimento de técnicas espectroscópicas que utilizam esta faixa de radiação eletromagnética esteve na obtenção de uma fonte capaz de gerar este tipo de radiação. Somente nos últimos 30 anos, com a construção e ampliação de aceleradores de elétrons denominados de anéis de luz síncrotron foi possível gerar radiação eletromagnética com alta intensidade na região do UV de vácuo aos raios-X duros ${ }^{2,3}$. Este avanço tecnológico consolidou as técnicas espectroscópicas de absorção de raios-X como uma das mais poderosas ferramentas na investigação da estrutura de materiais complexos.

Nestes aceleradores, os elétrons circulam em velocidades relativísticas (próximos da velocidade da luz) e, quando passam através de dispositivos magnéticos colocados no trajeto, ocorre alteração de sua órbita provocando a emissão de radiação eletromagnética, denominada luz síncrotron. A luz síncrotron possui características peculiares, como emissão intensa e alto brilho na região do UV aos raios$\mathrm{X}$ duros. Os aceleradores síncrotron ficam em laboratórios nacionais onde podem ser realizados diferentes tipos de experimentos, tais como medidas de absorção e de fluorescência de raios-X, cristalografia de proteínas, difração de filmes finos e espalhamento de raios-X a baixos ângulos (SAXS). O Brasil possui uma instalação deste tipo denominado Laboratório Nacional de Luz Síncrotron (LNLS), localizado no estado de São Paulo, na cidade de Campinas ${ }^{4}$.

*e-mail: morari@yahoo.com
Na linha de luz SGM ("Spherical Grating Monochromator") do LNLS nosso grupo vem realizando há três anos experimentos de absorção de raios-X na borda K do Nitrogênio (N K XANES), com o objetivo principal de caracterizar os estados de oxidação dos nitrogênios presentes em polímeros condutores da família da poli(anilina) livre (PANI, Esquema 1) ou confinada em matrizes inorgânicas. A importância na determinação dos estados de oxidação dos nitrogênios na PANI deve-se ao fato da estrutura eletrônica destes polímeros estar intimamente associada com o estado de oxidação dos nitrogênios presentes nas cadeias poliméricas ${ }^{5-7}$. As polianilinas são uma importante classe de polímeros condutores ${ }^{8}$ pois, além de serem dopadas facilmente por protonação, são relativamente mais estáveis ao ar e temperatura que os demais polímeros condutores, principalmente o poli(acetileno). Sua estrutura na forma base consiste de unidades repetitivas de segmentos reduzidos (benzenóides) e oxidados (quinóides) ${ }^{8}$, como representado no Esquema 1. Os termos "leucoesmeraldina" (PANI-LB), "esmeraldina" (PANI-EB) e "pernigranilina" (PANI-PB) referem-se aos diferentes estados de oxidação do polímero para $\mathrm{y}=1,0,5$ e 0 , respectivamente. A forma condutora da PANI é a forma sal ou protonada da PANI-EB, conhecida como sal de esmeraldina da PANI (PANI-ES, Esquema 1).

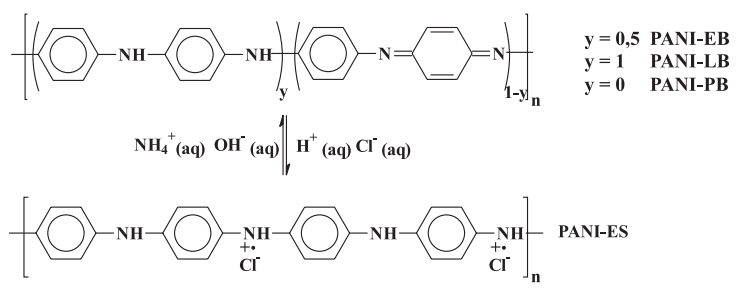

Esquema 1. Representação da estrutura da PANI nos seus diferentes estados de oxidação e na forma condutora esmeraldina sal

$\mathrm{Na}$ caracterização dos estados de oxidação do nitrogênio da PANI e de seus derivados foi necessário levantar um banco de dados, uma vez que não havia na literatura espectros de absorção de raios-X na borda $\mathrm{K}$ do nitrogênio para diferentes compostos, de 
modo a permitir uma análise dos espectros dos polímeros. Desta forma neste trabalho pretendemos ilustrar, com os dados obtidos para a PANI livre e os nanocompósitos de PANI com argila montmorilonita (MMT), a potencialidade da espectroscopia N K XANES na elucidação da estrutura eletrônica dos átomos de nitrogênio.

\section{PROCESSO DE ABSORÇÃO DE RAIOS-X}

É bem sabido que uma onda eletromagnética pode ser espalhada e/ou absorvida ao interagir com a superfície de um material. No caso da absorção, a intensidade (I) do feixe de raios-X sofre atenuação quando penetra no material sólido. Este decréscimo, é análogo à lei de Beer-Lambert ${ }^{9}$, isto é:

$\mathrm{I}(\mathrm{x})=\mathrm{I}_{\mathrm{o}} \exp (-\alpha \mathrm{x})$

mostrando que a intensidade diminui em função da penetração no material (x), uma vez que o argumento $(-\alpha \mathrm{x})$ da função é negativo. A uma certa distância $\mathrm{x}$, o decréscimo é tanto maior quanto maior for a magnitude do coeficiente de absorção $(\alpha)$. $O$ valor de $\alpha$ é função do material e do comprimento da onda eletromagnética. Os fótons na região do UV são fortemente absorvidos por todos os materiais sólidos, ao contrário dos raios-X duros $(\lambda<1 \mathrm{~nm})$ cujo coeficiente de absorção cresce com o aumento da densidade do material sólido e com número atômico dos elementos ${ }^{10}$. Portanto materiais formados de átomos pesados, como chumbo, absorvem mais do que os materiais formados de átomos leves, como berílio ${ }^{10}$.

A Figura 1 mostra esquematicamente um espectro de absorção característico - o coeficiente de absorção diminui com o aumento da energia do fóton incidente, porém existem variações bruscas, de modo que o espectro exibe um padrão característico de dente de serra $^{11}$. Estas variações correspondem às diferentes bordas de absorção presentes no material. A absorção de raios-X ocorre se a energia do fóton incidente for transferida para um elétron fortemente ligado ao átomo. Na Figura 2, por ex., representamos esquematicamente como seria a absorção de um elétron da camada K (nível 1s) de um átomo de um material sólido. Considere fótons com energia menor que o limiar de ionização $\left(h v_{1}\right)$, estes são fracamente absorvidos pelo material uma vez que não há estados de energia desocupados abaixo desta energia. Entretanto, quando a energia do fóton atinge o valor $h v_{2}$ ocorre um brusco aumento da absorção, correspondendo à borda de absorção da camada K, esta energia é chamada de limiar de ionização para o elétron $1 \mathrm{~s}^{10-12}$. Se a energia do fóton incidente continuar a aumentar $\left(h v_{3}\right)$ o elétron pode deixar o átomo, deixando-o ionizado. Este fato explica porque o coeficiente de absorção é muito similar à secção de choque do efeito fotoelétrico, i.e., há a probabilidade de ejeção de elétrons do átomo devido à absorção de fótons ${ }^{10-12}$. Se o valor de energia do fóton continuar a aumentar, a absorção começa a diminuir gradativamente, porém podem ocorrer novos saltos bruscos, desde que existam outras bordas de absorção no material.

De forma análoga à emissão de raios-X, a absorção de raios-X também pode ser explorada para detectar um elemento químico. Isto se deve ao fato dos valores das energias das bordas de absorção serem características de cada elemento químico. Compare, por ex., os elementos berílio e chumbo. O átomo de berílio possui massa atômica pequena, tendo somente quatro elétrons, ocupando os níveis 1s e 2s. Estes níveis possuem pequena energia de ionização, o limiar de absorção do nível 1s ocorre próximo de $111,5 \mathrm{eV}^{13}$, em comparação com os mesmos níveis no átomo de chumbo. Para maiores valores de energia, este mecanismo de fotoionização não é ativo, uma vez que o berílio não possui mais níveis que possam absorver esta energia de forma

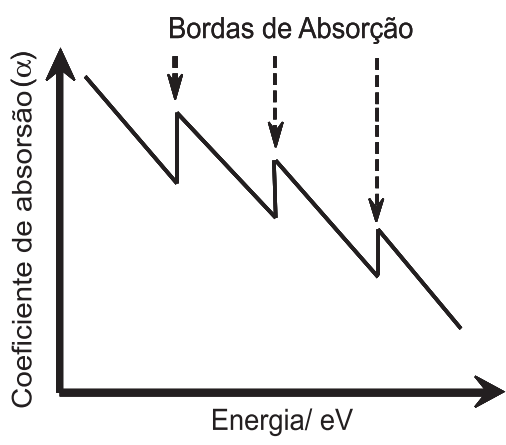

Figura 1. Representação esquemática da variação do valor do coeficiente de absorção de raios-X para um dado material em função da energia (ou comprimento de onda), exibindo um perfil característico de dente de serra

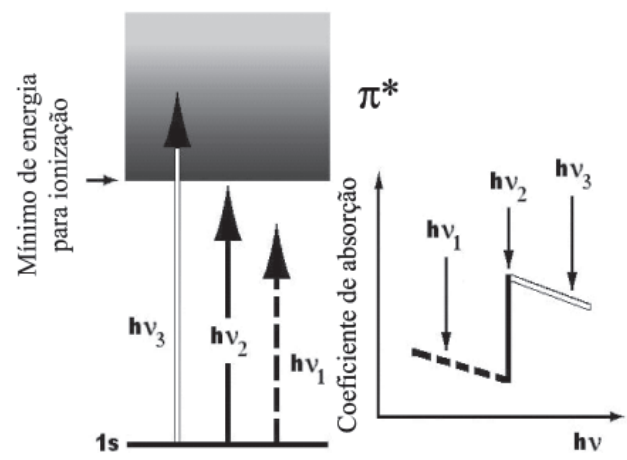

Figura 2. Representação esquemática das diferentes energias de fótons de raios$X$ comparadas com os limiares de ionização para um dado material. O fóton de energia $h v_{3}$ não é suficiente para produzir a ionização e não é absorvido. $O$ fóton de energia $h v_{2}$ possui o valor exato de energia da borda. A energia do fóton $\mathrm{hv}_{1}$ é mais que suficiente para produzir a ionização e dessa forma é absorvido. À direita, o espectro de absorção correspondente na região da borda $\mathrm{K}$

significativa. Assim, uma vez que a absorção de raios-X é pequena, berílio é um excelente material para fabricar janelas que transmitem raios-X. Por outro lado, o chumbo possui 82 elétrons, que ocupam as camadas K, L, M, N, e O. Algumas destas camadas possuem alta energia de ionização e, conseqüentemente, absorvem fótons com pequenos comprimentos de onda (da ordem de $0,014 \mathrm{~nm}$, isto corresponde a uma energia de $88 \mathrm{KeV})^{13}$. Isto explica porque o chumbo é um bom material para absorver raios-X.

A presença de um elemento químico em um material é apenas uma das informações químicas que é possível obter do espectro de absorção de raios-X. De fato, o fenômeno de absorção é muito mais complexo do que aparenta e, portanto, carrega muito mais informações do que a nossa abordagem fez supor. Podemos verificar que os espectros de absorção são complexos, possuindo um conjunto de oscilações que se estendem por uma grande faixa em valores de energia (dezenas de unidades de eV).

Na Figura 3 representamos um típico espectro de absorção de raios-X; têm-se uma absorção grande próxima da borda e um conjunto de oscilações que vão perdendo intensidade à medida que nos afastamos da borda de absorção. Este conjunto de oscilações é dividido em duas regiões, uma próxima da borda de absorção, e outra que vai além da borda. A estrutura próxima da borda compõe o chamado NEXAFS ("Near-Edge X-ray Absorption Fine Structure"), ou mais conhecido como XANES ("X-ray Absorption Near-Edge Structure"), a segunda região compõe o chamado EXAFS ("Extended X-ray Absorption Fine Structure"). 
A região XANES inclui uma faixa de energia antes da borda de absorção até o começo da região EXAFS. A definição de onde termina a região XANES e começa a região EXAFS é arbitrária, mas existe um certo consenso de que a região XANES se estende até 50 eV após a borda de absorção. A região EXAFS pode ser definida como o ponto onde o comprimento de onda do elétron ejetado é igual à distância entre o átomo absorvedor e os átomos vizinhos, esta região pode se estender até $1000 \mathrm{eV}$ após a borda de absorção ${ }^{14}$.

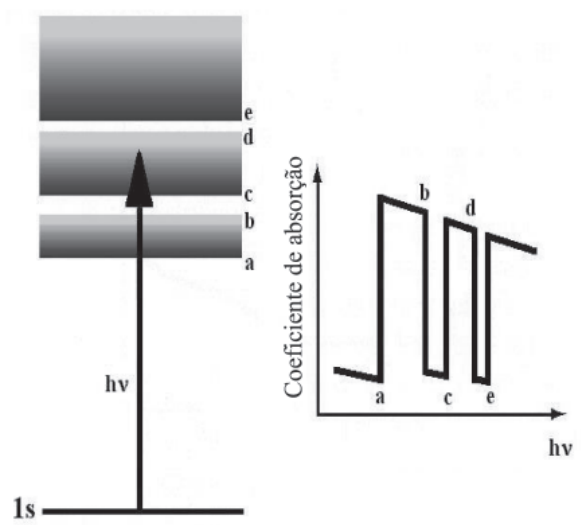

Figura 3. A distribuição de estados finais determina as variações no espectro de absorção. Neste exemplo, a banda dos estados finais é interrompida por gaps (b-c e d-e), que correspondem a decréscimos no espectro de absorção

O espectro EXAFS origina-se de efeitos de interferência ${ }^{14-16}$. A função de onda do elétron excitado se propaga para além do átomo e é parcialmente refletida pelos átomos vizinhos. A interferência entre a onda que se propaga e a que é refletida pelos átomos vizinhos causa as ondulações no espectro de absorção ${ }^{15,16}$. A interferência é construtiva ou destrutiva dependendo do comprimento de onda associado ao elétron e da distância interatômica. Assim, através do tratamento dos dados de EXAFS podem-se determinar as distâncias interatômicas entre o átomo que sofreu a excitação e os seus vizinhos próximos ${ }^{17,18}$.

O espectro XANES contém informação similar ao espectro EXAFS, mas a informação é mais difícil de extrair do ponto de vista matemático ${ }^{19,20}$. Isto é em grande parte devido às diferentes possibilidades de transições que podem ocorrer no sólido, o que na linguagem da teoria de espalhamento significa que na região XANES ocorrem espalhamentos múltiplos ${ }^{19,20}$. A intensidade da absorção é influenciada pelo número de elétrons que ocupam o estado inicial e que, portanto, podem participar da absorção, bem como depende da densidade de estados desocupados disponíveis e da probabilidade de transição ${ }^{19,20}$. As energias dos níveis desocupados dos átomos dependem do estado de oxidação e da natureza da ligação química que este átomo forma com seus vizinhos, tornando possível, através dos espectros XANES, diferenciar diferentes estados de oxidação deste elemento. A Figura 4 mostra um caso extremo, onde a banda de níveis desocupados tem interrupções ("gaps") de energia, provocando variações drásticas no coeficiente de absorção. Em casos menos extremos, não existem "gaps", mas variações moderadas na densidade de estados finais, produzindo modulação no espectro observado devido a diferenças no estado de oxidação e na natureza das ligações químicas dos materiais em estudo. A seguir nos concentraremos na análise da região XANES do espectro de absorção na borda $\mathrm{K}$ do nitrogênio para os diferentes compostos investigados.

\section{O EXPERIMENTO N K XANES NA LINHA SGM DO LNLS}

Para a realização dos experimentos N K XANES utiliza-se a

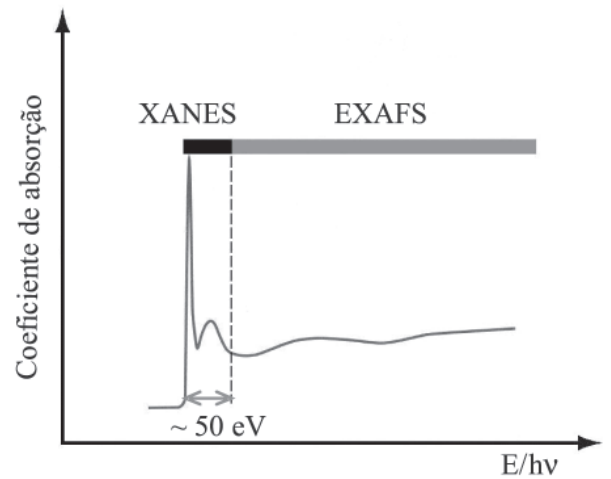

Figura 4. Espectro de absorção de raios-X genérico, mostrando as regiões características presentes no espectro

linha de luz SGM do LNLS, uma vez que esta linha pode funcionar na faixa de energia entre $250-1000 \mathrm{eV}^{21}$; esta faixa de energia engloba as bordas $\mathrm{K}$ do carbono, nitrogênio e oxigênio. As medidas de absorção são apenas possíveis em condição de ultra alto vácuo (a pressão dentro da câmara é de ca. $10^{-7}$ mbar), o que delimita o tipo de amostras passíveis de medição nestas condições. Podia-se imaginar inicialmente que as medidas de absorção fossem feitas pela razão de intensidade entre o feixe incidente $\left(\mathrm{I}_{\mathrm{o}}\right)$ e o feixe transmitido (I), como comumente se faz nas medidas de absorção na região do UV e visível. Entretanto, praticamente todas as substâncias absorvem fortemente na região do UV de vácuo, impedindo que a medida de absorção seja feita por transmissão do feixe pela amostra. A maneira como se faz a medida de absorção é conhecida como rendimento total de elétrons ("total electron yield") 22 . O que se mede é um sinal que é diretamente proporcional à quantidade de fótons absorvidos. Quando a amostra absorve ocorre emissão de elétrons (fotoelétrons, elétrons Auger, elétrons secundários), cuja intensidade é proporcional à quantidade de fótons absorvidos; para manter a amostra eletricamente neutra, faz-se o aterramento do compartimento de amostra, de modo que a corrente de reposição dos elétrons na amostra (corrente tipicamente da ordem de $10^{-12} \mathrm{~A}$ ) é proporcional à intensidade de fótons absorvidos. Podemos descrever sumariamente assim:

$\mathbf{I}_{\text {(co }}$ $\alpha \mathbf{I}$ $\alpha \mathbf{I}$ (fótons absorvidos)

Outra preocupação experimental é quanto ao número de amostras que se pode colocar de uma única vez no compartimento de medidas, uma vez que a cada substituição de amostra é necessária a quebra do vácuo e uma nova evacuação da câmara de amostras (tipicamente leva-se entre 3 a $5 \mathrm{~h}$ para chegar na pressão requerida para amostras pouco higroscópicas). O arranjo utilizado na linha SGM permite colocar para amostras sólidas na forma de pó até 40 amostras de uma vez. As amostras na forma de pó são fixadas sobre uma haste metálica contendo fita de carbono dupla face (Esquema 2).

Outras hastes sulcadas são colocadas sobre a haste principal para delimitar a área da amostra (ca. $\left.0,2 \mathrm{~cm}^{2}\right)$, bem como impedir a mistura de uma amostra com outra, uma vez que as medidas são feitas com a haste posicionada verticalmente na câmara da amostra.

\section{ESPECTROSCOPIA N K XANES}

Na literatura principalmente através dos trabalhos de Henning et $a l .{ }^{23}$ e Hitchcock et al. ${ }^{24}$ foi possível constatar a viabilidade do uso da técnica XANES na caracterização dos diferentes estados de oxidação dos nitrogênios para diferentes compostos aromáticos, podendo-se verificar também efeitos de conjugação, como a pre- 


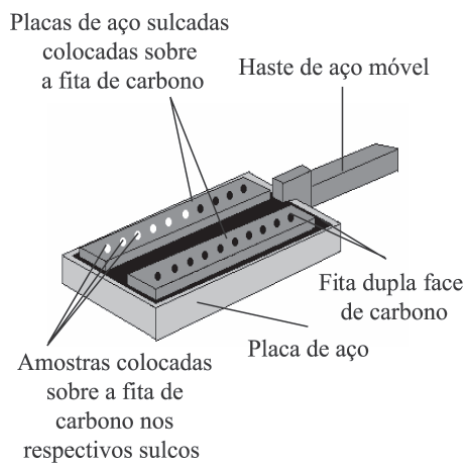

Esquema 2. Representação do suporte de amostra utilizado nas medidas $N$ $K X A N E S$ realizadas na linha SGM do LNLS

sença de estrutura quinóide em anéis benzênicos mono e di substituídos. Mais recentemente, estes autores demonstraram a viabilidade de se estudar os estados eletrônicos dos nitrogênios presentes nas cadeias da PANI. Henning et al. ${ }^{23}$ mostrou que o espectro $\mathrm{N} \mathrm{K}$ XANES da PANI é dominado por transições $1 \mathrm{~s} \rightarrow 2 \mathrm{p} \pi^{*}$ cujos valores de energia e intensidades são dependentes do estado de oxidação da PANI. O espectro N K XANES da PANI-EB (Esquema 1) é dominado por uma banda em 397,4 eV, que pode ser atribuída, através da comparação com o espectro N K XANES de um composto tomado como padrão (2-hidroxil-3-metoxi-benzilanilina), à transição $1 \mathrm{~s} \rightarrow 2 \mathrm{p} \pi^{*}$ do nitrogênio imínico $(=\mathrm{N}-)$. Foi observado que esta banda praticamente não está presente no espectro N K XANES

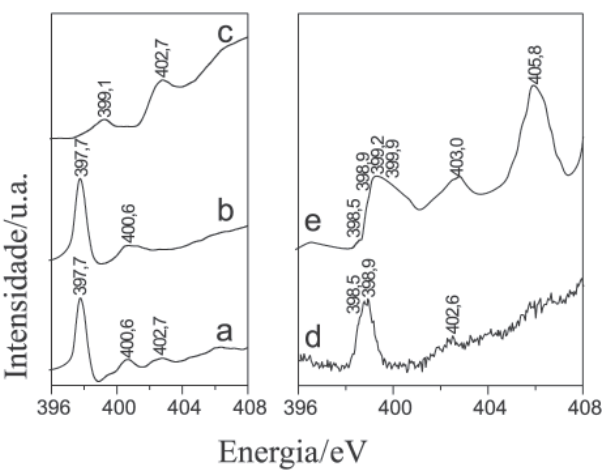

Figura 5. Espectro N K XANES das amostras de: PANI-EB (a), PANI-PB (b), e PANI-ES (c), poli(benzidina) (d), PANI-MMT (e)

da PANI-LB (forma totalmente reduzida da PANI, Esquema 1), demonstrando a consistência da atribuição feita. A presença de outras duas bandas observadas em 400,4 e 402,1 eV no espectro N K XANES da PANI-EB é devida a efeitos de conjugação, e foram atribuídas às transições $1 \mathrm{~s} \rightarrow 2 \mathrm{p} \pi^{*}$ dos nitrogênios imina deslocalizados e aminas, respectivamente.

Henning et al. ${ }^{23}$ obtiveram também o espectro N K XANES da PANI sintetizada eletroquimicamente com diferentes ácidos e verificaram que a intensidade da banda em 397,4 eV, que é dominante no espectro da PANI-EB, sofre significativa redução no espectro da PANI-ES, sendo que a intensidade desta banda depende do grau de protonação do polímero. Segundo estes autores, um segundo

Tabela 1. Valores das bandas, relacionadas com transições $1 \mathrm{~s} \rightarrow \pi^{*}$, observadas nos espectros N K XANES para diferentes compostos




efeito da protonação é a completa supressão da banda N1s $\rightarrow 2 \mathrm{p} \pi *$ (=N-), que é substituída por uma nova banda em 398,8 eV, atribuída ao nitrogênio imínico protonado.

A PANI-EB, PANI-PB e a PANI-ES preparadas quimicamente de acordo com o descrito por MacDiarmid et al. ${ }^{8}$ foram as primeiras amostras analisadas por nosso grupo na linha SGM do LNLS $^{25}$. Verifica-se no espectro da PANI-EB e PANI-PB (Figuras 5a e b, respectivamente) uma banda intensa em $397,7 \mathrm{eV}$, que pode ser atribuída aos nitrogênios imínicos, de forma semelhante à atribuição proposta por Henning et al. ${ }^{23}$. Fica evidente que esta banda não está presente no espectro da PANI-ES (Figura 5c), devido à protonação dos nitrogênios imínicos, a banda, que surge então em 399,1 eV, foi atribuída aos nitrogênios dos segmentos radicais cátion da PANI-ES. As outras bandas presentes no espectro da PANI-EB, PANI-PB e PANI-ES podem ser atribuídas às iminas deslocalizadas e aos nitrogênios amina (a Tabela 1 apresenta a atribuição tentativa).

O sucesso na obtenção e na análise dos espectros N K XANES da PANI permitiu estudar os espectros N K XANES dos nanocompósitos de PANI e derivados, preparados pela polimerização in situ dos monômeros dentro das cavidades da argila MMT. Entretanto, o espectro destes nanocompósitos apresentou bandas diferentes dos padrões de PANI livre, impondo a necessidade de obter um conjunto de espectros N K XANES de diferentes tipos de moléculas (Figura 6) contendo nitrogênios com distintas estruturas eletrônicas (Esquema 3). A Tabela 1 sumariza os dados obtidos, bem como a atribuição tentativa para as diferentes transições observadas. Verifica-se que a diferença entre os valores de energia muda muito pouco com o tipo de nitrogênio, porém é possível fazer uma correlação entre os valores obtidos e a estrutura dos diferentes compostos (Esquema 3). Deve-se mencionar que as bandas largas observadas em valores de energias maiores que ca. $404 \mathrm{eV}$ são devidas a transições do tipo $1 \mathrm{~s} \rightarrow \sigma^{*}$ cuja análise é mais complexa, uma vez que estas bandas são bem mais largas que as devidas às transições $1 \mathrm{~s} \rightarrow \pi^{*}$, dificultando a utilização destas bandas em uma análise comparativa. Apenas com a obtenção e análise destes dados foi possível determinar que as bandas em 398,5 e 398,9 eV presentes no espectro N K XANES da poli(benzidina) (Figura 5d) são devidos aos nitrogênios azo e fenazínicos, respectivamente ${ }^{25}$. Além disso, possibilitou comprovar que as bandas em 398,5 eV e 398,9 eV
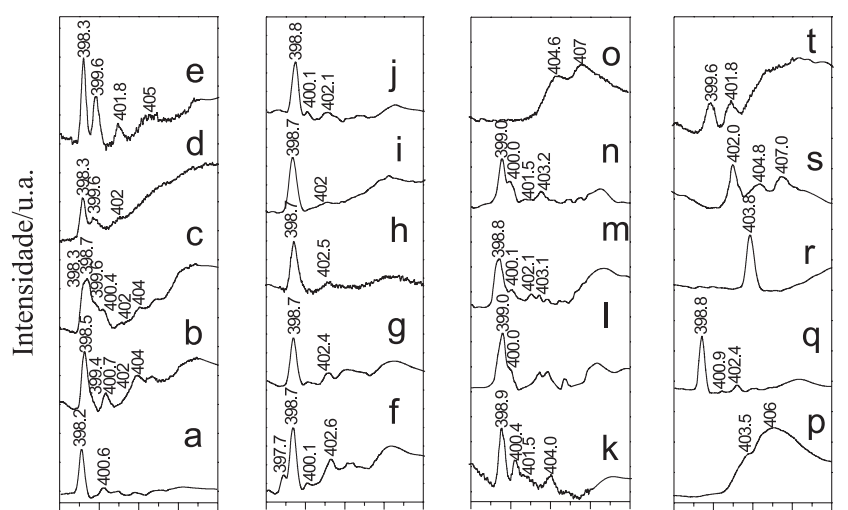

396400404408412396400404408412396400404408412396400404408412

\section{Energia/eV}

Figura 6. Espectros $N$ K XANES dos sólidos de: fenazina (a), fenosafranina (b), verde de Janus $B(c)$, azul do Nilo (d), oxazina (e), 4-amino-azobenzeno $(f), 4,4$ '-diamino-azobenzeno $(g)$, vermelho do Congo $(h)$, alaranjado de metila (i), vermelho de metila ( $j)$, amarelo de Titã ( $k)$, sudan III (l), naftol black $(m)$, red lake $C(n)$, cloreto de hidrazínio $(o)$, sulfato de hidrazínio $(p)$, 1,10-fenantrolina (q), ácido 3,5-dinitrobenzóico ( $r), N, N$ '-bifenil-1,4fenilenodiamina $(s)$, e violeta genciana $(t)$

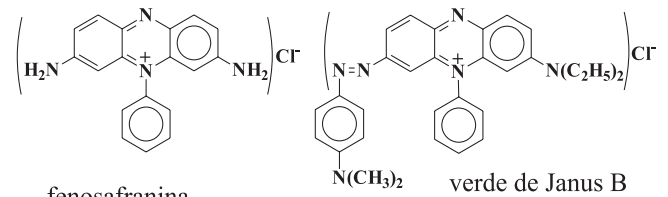

fenosafranina<smiles>CN(C)[13C](C)(C)O[Na]</smiles>

azul do Nilo<smiles>CCN(CC)c1ccc2nc3ccc(=[N+]=[N+]=O)cc-3oc2c1</smiles>

red lake $\mathrm{C}$

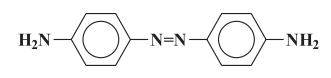

4,4'-diamino-azobenzeno

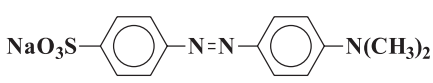

alaranjado de metila<smiles>CN(C)c1ccc(N=Nc2ccccc2C(=O)O)cc1</smiles>
vermelho de metila<smiles>CCCCOc1cc2cc([N+](=O)[O-])c(N=Nc3ccc([N+](=O)[O-])cc3)c(N)c2c(O)c1N=Nc1ccccc1</smiles>

naftol black

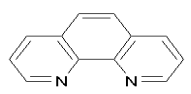

1,10-fenantrolina<smiles>Cc1ccc2nc(-c3ccc(I)cc3)sc2c1[N+](=O)[O-]</smiles>

violeta genciana

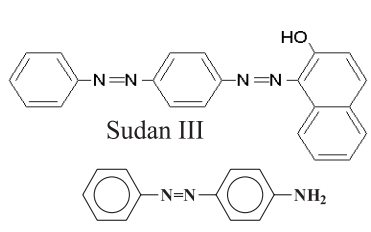

amino-azobenzeno

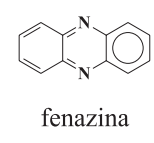

$\mathrm{ClO}_{4}$

Esquema 3. Representação das estruturas químicas dos compostos padrões

presentes no espectro N K XANES da PANI intercalada na argila Montmorilonita (Figura 5e) são devida aos nitrogênios azo e fenazínicos ${ }^{25}$.

Na Figura 7 é apresentado o resultado de experiência para verificar a existência de uma relação linear entre a intensidade do sinal XANES com a concentração de um determinado composto na amostra. Assim, realizaram-se medidas N K XANES para amostras constituídas pela mistura mássica de alaranjado de metila com $\mathrm{NaNO}_{3}$ para diferentes proporções mássicas entre os componentes; os espectros são apresentados na Figura 7. Estes compostos foram escolhidos, pois apresentam bandas intensas e bem separadas em valores de energia uma da outra. Verifica-se nitidamente a dependência do sinal com a concentração de um tipo de composto na amostra (Figura 7). Nota-se que até a razão mássica de alaranjado de metila/ $\mathrm{NaNO}_{3}$ igual a 3 temos um regime linear (Lei de BeerLambert $^{9}$ ), sendo o sinal XANES linearmente dependente da concentração de alaranjado de metila na amostra; acima deste valor 
tem-se um patamar, onde o sinal XANES não responde mais com a variação da concentração de alaranjado de metila na mistura sólida. Estes dados sugerem a possibilidade de utilização dos dados $\mathrm{N}$ $\mathrm{K}$ XANES com uma abordagem quantitativa.

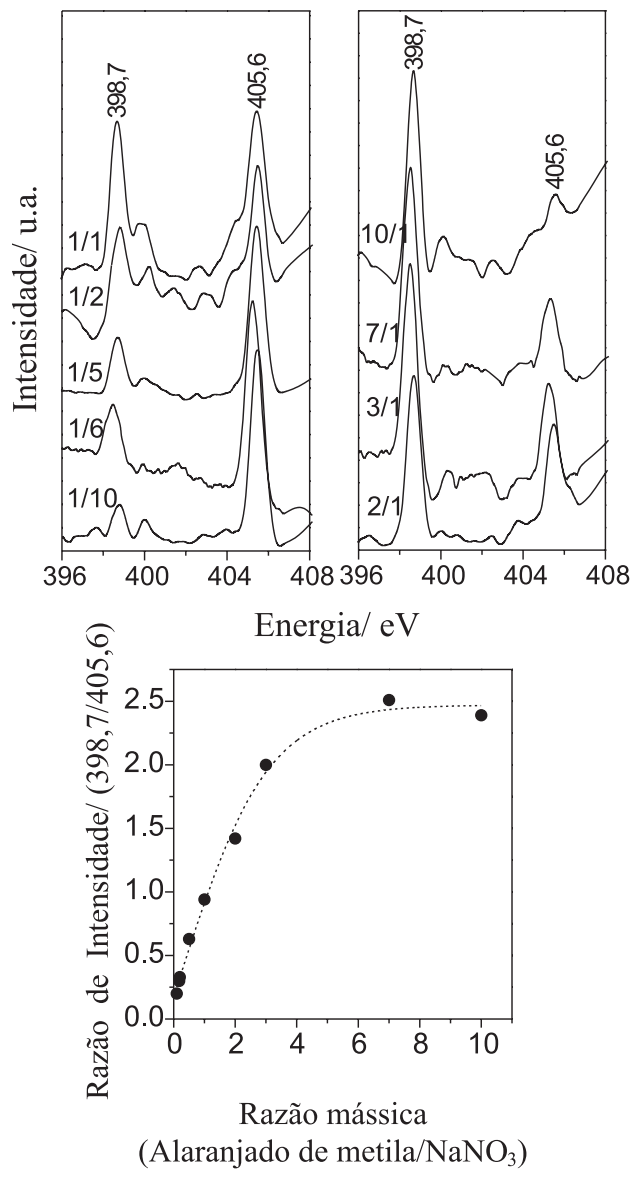

Figura 7. Espectros $N$ K XANES para diferentes misturas mássicas de alaranjado de metila/ $\mathrm{NaNO}_{3}$ (as proporções $\mathrm{m} / \mathrm{m}$ estão indicadas na figura) e gráfico representando a correlação entre a razão de intensidade das bandas 398,7 e 405,6 eV (transição 1s $\rightarrow \pi^{*}$ para os nitrogênios azo e do nitrato, respectivamente)

\section{CONCLUSÕES}

O espectro N K XANES é dominado pela presença de transições $1 \mathrm{~s} \rightarrow \pi^{*}$, quase sempre intensas e bem definidas em energia, permitindo correlacionar diretamente o valor de uma dada banda com o tipo de nitrogênio encontrado no material. Através de uma extensa coleção de espectros N K XANES obtidos na linha SGM do LNLS foi possível comprovar a presença de segmentos azo e fenazínicos na estrutura dos nanocompósitos de PANI-MMT e da poli(benzidina)-MMT.

\section{AGRADECIMENTOS}

À FAPESP pelo apoio financeiro e ao CNPq (M. L. A. Temperini). Os autores agradecem também ao LNLS pelas medidas N K XANES na linha SGM (proj. 1026/01, 1427/02, 1432/02 e 2169/03), bem como o auxílio do técnico da linha Sr. P. de Tarso durante a realização das medidas na linha SGM.

\section{REFERÊNCIAS}

1. Margaritondo, G.; Introduction to Synchrotron Radiation, Oxford University Press: New York, 1988, cap. 1, 3.

2. Bilderback, D. H.; Elleaume, P; Weckert, E.; J. Phys. B: At., Mol. Opt. Phys. 2005, 38, S773.

3. Heald, S. M. Em Chemical Analysis; Koningsberger, D. C.; Prins, R., eds.; John Wiley \& Sons: USA, 1988, vol. 92, cap. 4, p. 119.

4. Para maiores detalhes consulte a página eletrônica oficial do LNLS: http://www.lnls.br.

5. Shirakawa, H.; Angew. Chem., Int. Ed. 2001, 40, 2574.

6. MacDiarmid, A. G.; Angew. Chem., Int. Ed. 2001, 40, 2581.

7. Heeger, A. J.; Angew. Chem., Int. Ed. 2001, 40, 2591.

8. MacDiarmid, A. G.; Epstein, A. J.; Faraday Discuss. Chem. Soc. 1989, 88, 317; MacDiarmid, A. G.; Chiang, J. C.; Richter, A. F.; Sonosiri, N. L. D. Em Conducting Polymers; Alcácer, L., ed.; Reidel Publications: Dordrecht 1987, p. 105; MacDiarmid, A. G.; Epstein, A. J. Em Frontiers of Polymers and Advanced Materials; Prasad, P. N., ed.; Plenum Press: New York, 1984, p. 251.

9. Atkins, P. W.; Physical Chemistry; Oxford University Press: OxfordLondon, 1994, p. 545.

10. Margaritondo, G.; Elements of Synchrotron Light for Biology, Chemistry and Medical Research, Oxford University Press: New York, 2002, cap. 3, 72.

11. Margaritondo, G. Em ref. 1, cap. 2, p. 50.

12. Durham, P. J.; Em ref. 3, cap. 2, p. 53.

13. Thompson, A. C.; Vaughan, D.; X-ray Data Booklet Compiled and Edited by Lawrence Berkeley National Laboratory, Lawrence Berkeley National Laboratory University of California Berkeley: California 94720, $2^{\text {nd }}$ ed., 2001.

14. Bianconi, A.; Appl. Surf. Sci. 1980, 6, 392.

15. Lee, P. A.; Citrin, P. H.; Eisenberger, P.; Kincaid, B. M.; Rev. Mod. Phys. 1981, 53, 769 .

16. Stern, E. A.; Em ref. 3, cap. 1, 3.

17. Abruña H. D. Em Modern Aspects of Electrochemistry; Bockris, J. O’M.; White, R. E.; Conway, B. E., eds.; Plenum Press: New York, 1989, vol. 20, cap. 4, p. 265.

18. Parsons, J. G.; Aldrich, M. V.; Gardea-Torresdey, J. L.; Appl. Spect. Rev. 2002, 37, 187.

19. Bianconi, A.; Em ref. 3, cap. 11, p. 573.

20. Manne, R.; Åberg, T.; Chem. Phys. Lett. 1970, 7, 282; Manne, R.; Åberg, T. Em Benchmark Papers in Physical Chemistry and Chemical Physics/2: $X$-ray Photoelectron Spectroscopy; Carlson, T. A., ed.; Dowden, Hutchinson \& Ross, Inc.: Stroudsburg-Pennsylvania 1978, cap. 3, p. 124.

21. A resolução da linha é de ca. $0,1 \mathrm{eV}$. Os espectros N K XANES obtidos na linha SGM foram calibrados utilizando-se para a primeira banda de absorção do espectro N K XANES do $\mathrm{KNO}_{3}$ o valor de 405,5 eV, segundo a ref. Vinogradov, A. S.; Akimov, V. N.; Opt. Spectrosc. 1998, 85, p. 53.

22. Heald, S. M. Em ref. 3, cap. 3, p. 87

23. Hennig, C.; Hallmeier, K. H.; Bach, A.; Bender, S.; Franke, R.; Hormes, J.; Szargan, R.; Spectrochim. Acta, Part A 1996, 52, 1079; Pavlychev, A. A.; Hallmeier, K. H.; Hennig, C.; Hennig, L.; Szargan, R.; Chem. Phys. 1995, 201, 547; Hennig, C.; Hallmeier, K. H.; Szargan, R.; Synth. Met. 1998, 92, 161.

24. Francis, J. T.; Hitchcock, A. P.; J. Phys. Chem. 1992, 96, 6598.

25. Do Nascimento, G. M.; Izumi, C. M. S.; Constantino, V. R. L.; Temperini, M. L. A.; Activity report of Brazilian Synchrotron Light Laboratory 2002 2003, p. 141; Do Nascimento, G. M.; Constantino, V. R. L.; Temperini, M. L. A.; J. Phys Chem. B 2004, 108, 5564; Do Nascimento, G. M.; Constantino, V. R. L.; Landers, R.; Temperini, M. L. A.; Macromolecules 2004, 25, 9373. 